\title{
A New Model of Genetic Variation and Evolution Evaluates Relative Impacts of Background Selections and Selective
}

\section{Sweeps}

\author{
Xun $\mathrm{Gu}$ \\ Depatment of Genetics, Development and Cell Biology, Iowa State University, IA50011, \\ USA \\ Correspondence: xgu@iastate.edu
}




\section{Abstract}

Intra-population genetic variation and interspecies divergence in chromosome regions can be considerably affected by different local recombination rates. There are two models: $(i)$ the selective sweeps that reduces the genetic diversity at linked sites and elevates the divergence rate; and (ii) the background selection that reduces the genetic diversity at linked sites and divergence rate. An intriguing question, yet highly controversial, is which one is dominant. In this paper, I develop a framework of generalize background selection, formulated by a diffusion model with two killing functions: the one associated with (negative) background selection is the rate to stop a fixation process of a mutation randomly, and the other associated with positive background selection (selective sweep) is the rate to stop a loss process of a mutation randomly. A simple relationship between the level of reduced diversity and the rate of divergence is derived, depending on the strength of generalized background selection $(G)$ and the proportion of positive background selection $(\beta)$. We analyzed the interspecies divergence and intra-population diversity in low-recombination regions of three organisms (fruitfly, soybean and human). Strikingly, all datasets demonstrated the dominance of (negative) background selection, and the positive background selection (selective sweeps) only has a small contribution $(\beta \sim 10 \%)$. However, our analysis rejects the notion of $\beta=0$, namely, a complete negative background selection is unlikely. These findings may shed some lights on the long-term debates around Neutral Theory. 


\section{Introduction}

Levels of intra-species genetic variation and rates of molecular evolution in different regions of the genome may be greatly affected by differences in the rate of recombination $(1,2)$. Maynard Smith and Haigh (3) proposed the hitchhiking hypothesis, where the spread of a favorable mutation reduces the level of neutral or nearly-neutral variability at linked sites; a process also termed 'selective sweeps' (4). On the other hand, Charlesworth et al. (5) argued that reduction of genetic variation in low-recombination regions can operate through the removal effects of purifying selections on deleterious mutations, a process called 'background selections'. One may see a number of comprehensive reviews for a rich body of literatures in both theoretical an empirical studies (6-11).

The advent of high throughput genomics and the long-standing selectionistneutralist debate $(12,13)$ have inspired tremendous attempts to infer the relationship between the level of intra-species diversity, the local recombination rate and the interspecies divergence $(14,15)$. Pioneered by Kaplan et al. (16), Wiehe and Stephan (17) and Kim and Stephan (18), most studies concluded that considerable reduction in genetic diversity was the result of joint effects of selective sweeps and background selections (19-23). By contrast, low-recombination chromosome regions generally showed no considerable reduced or elevated interspecies divergence (24-27). Because background selection predicts a much lower interspecies divergence in low-recombination regions whereas selective sweep predicts a much higher one, this observation has been widely interpreted as the existence of selective sweeps under the background selections. Therefore, an intriguing question is which of these two nonexclusive causal factors is more dominant $(7,28,29)$; yet, the estimates differ substantially among different studies (35-41).

Herein, I attempt to formulate a new evolutionary framework called generalized background selection (Table 1), which includes two major types: (i) negative background selection, exemplified by the conversional background selection at closely-linked sites (5); and (ii) positive background selection, exemplified by the selective sweep at closelylinked sites (3). A new diffusion-limit model with two killing function (42) is then developed: the killing function for negative background selection measures the rate for 
the stochastic trajectory of a fixation process of a mutation to be randomly stopped, whereas the killing function for positive background selection measures the rate for that of a loss process of a mutation to be randomly stopped. The relationship between intrapopulation diversity and interspecies divergence is then derived, which can be applied to analyze the patterns of inter-species divergence and intra-species diversity in lowrecombination regions, while the recombination rate is treated as a biological variable underlying the strength of killing functions. The current study focuses on two fundamental issues: first, which one, selective sweeps or (negative) background selection, is more dominant $(7,24,27-29,43)$; and second, whether either one of them is sufficient to explain the observed diversity and divergence pattern (35-41).

\section{Results}

\section{Diffusion model under the generalized background selection}

A conceptual framework of generalized background selection is introduced as follows (Table 1). (i) Negative background selection: for instance, purifying selection against deleterious mutations may reduce intra-species diversity and interspecies divergence at closely-linked sites $(5,44-47)$. Since deleterious mutations are prevalent in the genome, many authors argued that (negative) background selection should be part of the basic model of genome variation and evolution (26, 27, 48, 49). (ii) Positive background selection: for instance, neutral or nearly neutral mutations can be rapidly fixed by a few favorable mutations in the surrounding chromosome region (selective sweeps), resulting in a considerable reduction of genetic variation (3) and an elevated rate of molecular evolution at closely-linked sites. Stephan (11) reviewed different inference methods that have been developed to detect selective sweeps and to localize the targets of directional selection in the genome.

The diffusion-limit model with two killing functions (42) is utilized to model the effects of generalized background selection. Let $x$ be the initial frequency of a mutation in the population. (i)The killing function associated with negative background selection, denoted by $k^{-}(x)$, is the rate for the stochastic trajectory of a fixation process to be randomly stopped, decreasing the fixation probability of a mutation. (ii) The killing function associated with positive background selection, denoted by $k^{+}(x)$, is the rate for 
the stochastic trajectory of a loss process to be randomly stopped, decreasing the loss probability of a mutation from the population (or, indirectly, increasing the fixation probability). In the current study, both killing functions are specified in the form of $k^{-}$ $(x)=b x(1-x)$ and $k^{+}(x)=h x(1-x)$, where $b$ and $h$ are the coefficients of negative and positive background selections, respectively. These forms of killing functions are not only mathematically convenient, but also reflect the fact that the generalized background selection is effective for mutations with low frequencies.

Let $u(x)$ be the fixation probability of a mutation with the initial frequency $x$. As shown in Materials and Methods, the Kolmogrov backward equation of $u(x)$ with two killing functions specified above is given by

$$
\frac{\sigma^{2}(x)}{2} \frac{d^{2} u}{d x^{2}}+\mu(x) \frac{d u}{d x}-\left[k^{-}(x)+k^{+}(x)\right] u+k^{+}(x)=0
$$

with boundary conditions $u(0)=0$ and $u(1)=1$. The mean $\mu(x)$ and variance $\sigma^{2}(x)$ parameters can be determined under the Wright-Fisher model (see below).

Next we consider intra-species genetic diversity. In the case of no overdominance, any mutation that appears in a finite population is either ultimately lost or fixed. The effects of generalized background selection make the maintenance of intraspecies diversity difficult, because both killing functions tend to reduce the genetic diversity by increasing the chance of a mutation to be either fixed or lost. Nevertheless, under the steady flux of new mutations over many generations, a balance will be reached between production of new mutants and their random loss or fixation. Under this statistical equilibrium there is a stable frequency distribution at different sites in which mutations are neither fixed nor lost (50). Denote the stable frequency of mutations by $p$. Given the initial frequency $x$, let $J(x)=E[2 p(1-p)]$ be the expected heterozygosity of a nucleotide site. As shown in Materials and methods, the steady-flux model with two killing functions claims that $J(x)$ satisfies the following backward equation

$$
\frac{\sigma^{2}(x)}{2} \frac{d^{2} J}{d x^{2}}+\mu(x) \frac{d J}{d x}-\left[k^{-}(x)+k^{+}(x)\right] J+2 x(1-x) v=0
$$

where $v$ is the mutation rate; the boundary conditions of Eq.(2) are $J(0)=J(1)=0$. 


\section{Neutral interspecies divergence under generalized background selection}

An intriguing question is to what extent the prediction of the neutral theory, i.e., the rate of neutral evolution $(\lambda)$ equals to the mutation rate $(v)$, could be affected by the effects of (negative) background selections and selective sweeps $(41,51-53)$. To this end, we obtained the fixation probability $u(x)$ given by solving Eq.(1) under the neutral model (see Materials and Methods). Note that the rate $(\lambda)$ of molecular evolution is defined by the amount of new mutations $2 N_{e} v$ multiplied by the fixation probability $u(x)$ with the initial frequency $x=1 / 2 N_{e}$; where $N_{e}$ is the effective population size. Let $H=4 N_{e} h$ be the intensity of positive background selection, $B=4 N_{e} b$ be the intensity of negative background selection, and $G=B+H$ be the intensity of generalized background selection, respectively (Table 1). Putting together, one can show

$$
\lambda=v\left[(1-\beta) \frac{2 \sqrt{G} e^{-\sqrt{G}}}{1-e^{-2 \sqrt{G}}}+\beta \sqrt{G}\left(\frac{1+e^{-2 \sqrt{G}}}{1-e^{-2 \sqrt{G}}}\right)\right]
$$

where $\beta=H /(B+H)=h /(b+h)$ is the proportion of positive background selection (selective sweeps), or 1- $\beta$ is that of negative background selections. Eq.(3) shows that, in addition to the mutation rate $(v)$, the rate of neutral rate $(\lambda)$ can be affected by the strength of generalized background selection $(G)$ and the proportion of positive background selection $(\beta)$. While the first part on the right hand of Eq.(3) is the rate component associated with negative background selection, the second one is that associated with positive background selection. It appears that Eq.(3) is reduced to $\lambda=v(54)$ when $G=0$.

Fig.1 panel A presents the plotting of the (neutral) rate-mutaion ratio $\lambda / v$ against the strength $(G)$ of generalized background selection under various proportion $(\beta)$ of positive background selection. (i) When $\beta=0$ (i.e., no positive background selection, and so $G=B)$, the rate of neutral evolution $(\lambda)$ is always less than the mutation rate $(v)$; for instance, $\lambda$ is about $85 \%, 55 \%$ and $27 \%$ of the mutation rate (v) when $B=1,4$ and 10 , respectively. Indeed, a very strong negative background selection would virtually cease the neutral divergence between species, i.e., $\lambda \approx 0$. (ii) By contrast, when $\beta=1$ (i.e., complete positive background selection, and so $G=H)$, the rate $(\lambda)$ of neutral evolution is always larger than the mutation rate $(v)$; for instance, $\lambda$ is about $131 \%, 204 \%$ and $316 \%$ 
of the mutation rate $(v)$ for $H=1,4$ and 10 , respectively. When $H>1$, the rate of neutral evolution is asymptotically linear with the squared root of $H$. (iii) In the general case of $0<\beta<1$, a numerical analysis of Eq.(3) indicates a critical value $\beta_{c} \approx 0.334$. When $\beta<\beta_{c}$, a weak positive background selection, the neutral rate decreases from $\lambda=v$ at $G=0$ with the increasing of $G$ until approaching to a minimum; since then $\lambda$ increases with the increasing of $G$, ultimately toward $\lambda>v$. On the other hand, when $\beta>\beta_{c}$, an intermediate or strong positive background selection, the neutral rate $\lambda$ increases with the increase of $G$ such that $\lambda>v$ always holds.

\section{Neutral genetic variation under generalized background selection}

In contrast to the interspecies divergence, both negative and positive selections contribute to the reduction of intra-species genetic diversity (Table 1), which can be derived as follows. One can solve Eq.(2) to obtain the expected neutral heterozygosity of a site, $J(x)$, in the case of neutrality, i.e., $\mu(x)=0$ and $\sigma^{2}=x(1-x) / 2 N_{e}$ (Materials and Methods). As the expected neutral genetic diversity $(\pi)$ of a site is given by $\pi=2 N_{e} J(x)$ at $x=1 / 2 N_{e}$, we show that the expected neutral genetic diversity under the generalized background selection is given by

$$
\frac{\pi}{\pi_{0}}=\left(\frac{2}{\sqrt{G}}\right)\left(\frac{1-e^{-\sqrt{G}}}{1+e^{-\sqrt{G}}}\right)
$$

where $\pi_{0}=4 N_{e} v$ is the neutral genetic diversity without generalized background selection. It appears that the ratio of neutral genetic diversity $\left(\pi / \pi_{0}\right)$ depends on a single parameter $G$, the strength of generalized background selection; $\pi / \pi_{0}<1$ always holds, and $\pi / \pi_{0}=1$ only when $G=0$ (Fig.1 panel B). Since $G=4 N_{e} g$, where $g=b+h$ is the coefficient of generalized background selection, Eq.(4) indicates that reduction of intra-population neutral diversity by the generalized background selection becomes severe in a large population (in a scale of the squared root of $N_{e}$ ), whereas this reduction can be compromised by genetic drifts in a small population. 


\section{Statistical analysis of chromosome regions with low recombination}

A number of studies $(26,27,33,34,55)$ indicated a dominant role of (negative) background selection on the reduction of genetic diversity in regions with low recombination. However, it remains highly controversial whether it is sufficient to explain the observed intra-population diversity and interspecies divergence, without invoking selective sweeps. It appears that Eq.(3) and Eq.(4) together provide a straightforward procedure to address this key issue. Fig. 2 shows the $\lambda / v-\pi / \pi_{0}$ curve for different values of $\beta$, the proportion of positive background selection. Impressively, for genes with considerable reduced genetic diversity, say, $\pi / \pi_{0}<0.5$, the degree of interspecies divergence $(\lambda / v)$ is highly sensitive to the value of $\beta$.

\section{Statistical procedure}

By contracting chromosome regions with low recombination to those with free recombination, one can design a simple computational procedure to estimate $G$ and $\beta$. Suppose that we have two sets of genes: (i) genes are located in chromosome regions with free recombination, with the neutral intra-species diversity denoted by $\pi_{0}$, and the interspecies neutral divergence denoted by $d_{S 0}$. And (ii) genes are located in chromosome regions with low recombination, with neutral intra-species diversity denoted by $\pi$, and the interspecies neutral divergence denoted by $d_{S}$. After calculating the $\pi / \pi_{0}$ ratio, the strength of generalized background selection $(G)$ for genes in low-recombination regions is obtained by numerically solving Eq.(4). Next, one can calculate the divergence ratio $d_{S} / d_{S O}$ as a proxy to the $\lambda / v$ ratio in Eq.(3), allowing to estimate $\beta$ after replacing the parameter $G$ by its estimate.

\section{Non-crossover regions (NC) of D. melanogaster}

Campos et al. (27) used next-generation DNA sequence data of a population of $D$. melanogaster to compare the intra-population diversity and interspecies divergence across the whole genome. They analyzed 268 genes located in five independent heterochromatic regions that lack crossover ('non-crossover regions', NC) of $D$. melanogaster, contrasting to genes located in the crossover regions (AC short for 
autosomes and $\mathrm{XC}$ for $\mathrm{X}$-chromosome). For each gene group, the mean sequence divergence between D. melanogaster and D. yakuba was calculated.

We reanalyzed Campos et al. (27)'s data by computing the ratio $\pi / \pi_{0}: \pi$ is from synonymous diversity in each of five NC regions (and the pooled), and $\pi_{0}$ is from that in $\mathrm{AC}$ or XC, respectively. According to Eq.(4), it is straightforward to estimate $G$, the strengths of generalized background selection. Table 2 shows that the estimate of $G$ ranges from $0.40 \times 10^{2} \sim 12.2 \times 10^{2}$. This variation of estimated $G$ may reflect different recombination rates among NC regions, but the $95 \% \mathrm{CI}$ (confident interval) of estimated $G$ is broad. Next we estimated $\beta$ (the proportion of positive background selection) by Eq.(3), where the ratio $\lambda v$ is calculated by the interspecies synonymous distances in NC regions and $\mathrm{AC} / \mathrm{XC}$ regions, respectively (Table 2). It is impressive that the range of estimated $\beta$ among five NC regions is narrow, ranging from $2.48 \%$ to $15.1 \%$.Moreover, we used the pooled $\mathrm{NC}$ data to statistically test the null hypothesis of no positive background selection $(\beta=0)$. In the case $\mathrm{AC}$ as crossover regions, the estimated $\beta=8.06 \%$, with $95 \%$ CI from $3.51 \%$ to $12.0 \%$, and virtually the same results in the AC case (Table 2). Therefore, the pooled NC region analysis suggests that, to explain the pattern of genetic diversity and divergence, a weak but significant positive background selection (selective sweeps) in NC regions is required.

\section{Pericentromeric regions in Soybean (Glycine max)}

Du et al. (43) calculated synonymous distances $\left(K_{S}\right)$ of genes between soybean (Glycine max) and its annual wild relative, Glycine soja. They compared the mean $K_{S}$ $\left(K_{S, a r m}\right)$ located in a chromosomal arm (high recombination rate) and those $\left(K_{S, p e r i}\right)$ in a pericentromeric region (low recombination rate) in three genomic datasets: $(i)$ highconfidence genes annotated in the soybean reference genome; (ii) singletons (single-copy genes) from high-confidence genes; and (iii) WGD (whole genome duplication) duplicate pairs, each of which has one copy in a chromosomal arm and the other one in a pericentromeric region. The ratio $K_{S, p e r i} / K_{S, a r m}$ is $0.820,0.764$, and 0.731 for the three datasets, respectively. It has been roughly estimated that the ratio of synonymous diversity in pericentromeric regions to chromosomal arms within soybean population, denoted by $\pi_{S, p e r i} / \pi_{S, \text { arm }}$, is about 0.19 (56). In this case, one may obtain the estimate of 
strength of generalized background selection $(G)$ as 110.3, and the estimates of the proportion of positive background selection $(\beta)$ as $7.73 \%, 7.14 \%$, and $6.81 \%$ for three datasets, respectively.

\section{Empirical relationship of $G$ with the rate of recombination $(r)$}

Numerous analyses have established a well-known positive correlation between the genetic diversity $(\pi)$ and the recombination rate $(r)(6-11,14)$. When the current model is applied to chromosome regions with different $r$ values, we expect an inverse relationship between the strength of generalized background selection $(G)$ and the recombination rate, as demonstrated by Eq.(4) that genetic diversity $(\pi)$ is inversely determined by $G$ (Fig.1 panel B).

We analyzed the inter-species divergence and intra-species variation in different human chromosome regions with different recombination rate measured by $\mathrm{cM} / \mathrm{Mb}$; data from Nachman (24). Fig.3 panel A shows the mean strength of generalized background selection $(G)$ in genes located in low $(\mathrm{cM} / \mathrm{Mb}<1)$, middle $(1<\mathrm{cM} / \mathrm{Mb}<2)$ and high $(\mathrm{cM} / \mathrm{Mb}>2)$ recombination regions, respectively. A strong generalized background selection in low recombination regions has been observed. Meanwhile, Fig.3 panel B shows the mean proportion of positive background selection $(\beta)$ in genes located in low, middle and high recombination regions, respectively. Interestingly, the estimate of $\beta=8.9 \%$ in human low recombination region is very similar to that in fruitfly $(\sim 8 \%)$ or soybean $(7 \%-8 \%)$.

Kim and Stephan (18) showed that for the chromosome region with low recombination rate, the joint effects of deleterious and beneficial mutations on neutral variation can be approximated by

$$
\frac{\pi}{\pi_{0}} \approx \frac{f r}{r+b f \alpha \mu}
$$

where $r$ is the local recombination rate, $\alpha$ is the (positive) selection intensity, $\mu$ is the rate of adaptive substitution, $f$ describes the reduction of $N_{e}$ owing to deleterious mutations, and $b$ is an empirically-determined constant. While the theoretical derivation of the $G-r$ inverse relationship remains challenging, one can establish an empirical one by equating Eq.(5) with Eq.(4); under the assumption of $G>1$, it is approximated by 


$$
\sqrt{G} \approx 2\left(\frac{1}{f}+\frac{b \alpha \mu}{r}\right)
$$

Indeed, Fig.3 panel $\mathbf{C}$ shows a rough linear relationship between the squared-root of $G$ and the inverse of $r$; the coefficient of correlation is $0.73, P$-value $<0.01$.

\section{Discussion}

Based on the diffusion-limit model with two killing functions, I have formulated a framework of generalized background selection that has two predictions: $(i)$ reduction of intra-species neutral diversity is inversely determined by a single parameter $(G)$, the strength of generalized background selection that combines both negative or positive effects; and (ii) the rate of neutral divergence between species decreases with $G$, but increases with the proportion $(\beta)$ of positive background selection. Many studies attempted to determine the relative impact of selective sweeps to (negative) background selections on closely-linked sites. For instance, Pouyet et al. (33) concluded that the negative background selection influences as much as $85 \%$ of the genetic variants of the human genome, and Campos et al. (27) claimed that a strong selective sweep was unlikely in non-crossover regions of Drosophila. Our case-studies in three organisms (fruitfly, soybean and human) showed that the proportion $(\beta)$ of positive background selection (selective sweeps) in chromosome regions with low recombination rate is statistically significant, though on average, less than $10 \%$.

Compared to previous work (6-11), the new model may have some advantages.

First, it provides a straightforward approach to data analysis without oversimplification about the selection themes, as shown by Eqs.(3) and (4). The role of recombination rate $(r)$ is elaborated by its inverse relationship with the strength of generalized background selection $(G)$. Second, the new model provides a biologically intuitive explanation why the mechanism of selection sweeps is essential. Suppose $G=167$ as estimated from the (pooled) NC regions of Drosophila. If the (negative) background selection is the only mechanism underlying the reduced genetic diversity, i.e., $\beta=0$, the rate of neutral divergence between species calculated by Eq.(3) would be as low as $10^{-5}$ of that in crossing-over regions, that is, the Muller's ratchet (57) virtually ceases the pace of 
evolution. Surprisingly, if we assume $\beta=0.02$, i.e., a very small portion of selective sweeps, the rate of neutral divergence is only as low as $26 \%$ of that with free recombination (Fig.1 panel A). Third, one may design a new Bayesian approach for the detection of sites targeted by positive selection: for a given chromosome region with dramatically reduced diversity, a high level of sequence divergence between closelyrelated species may indicate the role of selective sweeps. Finally, our analysis may shed some lights on the recent debates on Neutral Theory $(12,13)$. If the proportion $(\beta)$ of selective sweeps estimated from chromosome regions with low recombination is generally applicable to the whole genome, one may conclude that neutral or nearly neutral selection dominates the genome-wide variation and evolution, while approximately $8 \%$ of mutations may be subject to positive selection.

An immediate extension of the current analysis is to include non-neutral sites such as nonsynonymous sites $(27,33,43,51)$. It has been shown that the interplay between $G, \beta$ and $S$ (the strength of 'direct' selection on the site under study) reveals more sophisticated evolutionary scenarios ( $\mathrm{Gu}$, unpublished results). There are several challenges remaining. First, the detailed structure of the inverse relationship between $G$ and the recombination rate is desirable for both theoretical and empirical studies. Second, as time-dependent $N_{e}$ changes may affect the estimation of selection sweeps $(7,9,11,20$, 41 ), the effect of constant $N_{e}$ assumed in the current model needs to be investigated. Third, it has been shown that the MacDonald-Kreitman (MK) test could be affected by both background selection and selective sweeps (61). An interesting question is to what extent the key parameters $(G$ and $\beta$ ) may influence the MK test. And forth, several factors, such as GC content and biased gene conversion, can also influence the reduced intra-population genetic reduction $(25,58,59)$. It is important to remove those factor before the current method is applied. We will address these challenges in the future study, with the help of extensive computer simulation studies such as SLim (60).

\section{Materials and Methods}

\section{Datasets}

We used the genome-wide genetic diversity profiles of $D$. melanogaster provided by Campos et al. (27). They compiled 268 genes, which located in five independent 
heterochromatic regions that lack crossover ('non-crossover regions', NC) of $D$. melanogaster, contrasting to the crossover regions (AC short for autosomes and $\mathrm{XC}$ for $\mathrm{X}$-chromosome). For each chromosome region under study, the sequence divergence with D. yakuba is also calculated.

Soybean (Glycine max) was domesticated from its annual wild relative, Glycine soja, about 5000 years ago. One striking feature of the soybean genome is that $\sim 57 \%$ of the genomic sequence occurs in recombination-suppressed heterochromatic regions surrounding centromeres (referred to as pericentromeric regions). We used three genomic datasets compiled by Du et al. (43): (i) high-confidence genes (27571) annotated in the soybean reference genome; (ii) 12,994 singletons (single-copy genes) from highconfidence genes; and (iii) 2439 WGD (whole genome duplication) duplicate pairs, each of which is composed of one copy in a chromosomal arm and the other one in a pericentromeric region.

Human dataset including 17 genes are obtained from Nachman (24) for which the sample size is greater than ten. For each gene, the genetic diversity $(\pi)$ in the human population, the sequence divergence with chimpanzees, and the recombination rate (in the human genome, measured by $\mathrm{cM} / \mathrm{Mb}$ ) are available.

\section{Kolmogrov backward equations with two killing functions}

\section{Fixation probability}

Let $u(x)$ be the probability of an allele to be ultimately fixed in the population, given the initial allele frequency $(x)$. By the standard diffusion theory, $u(x)$ satisfies the following Kolmogrov backward equation

$$
\frac{\sigma^{2}(x)}{2} \frac{d^{2} u}{d x^{2}}+\mu(x) \frac{d u}{d x}=0
$$

with the boundary conditions $u(0)=0$ and $u(1)=1$. Second we consider the backward equation of $u(x)$ with a killing function associated with the negative background selection, $k^{-}(x)$, which is the rate for the stochastic trajectory of fixation process to be randomly stopped. It appears that $k^{-}(x)$ decreases the fixation probability of a neutral or nearlyneutral mutation. Karlin and Taylor (42) showed that in this case $u(x)$ follows 


$$
\frac{\sigma^{2}(x)}{2} \frac{d^{2} u}{d x^{2}}+\mu(x) \frac{d u}{d x}-k^{-}(x) u=0
$$

Next we consider the backward equation of $u(x)$ with a killing function $k^{+}(x)$ associated with the positive background selection. Let $u^{*}(x)=1-u(x)$ be the ultimate loss probability of an allele. The killing function $k^{+}(x)$ is then defined by the rate for the stochastic trajectory of loss process to be randomly stopped. Hence, $k^{+}(x)$ tends to decrease $u^{*}(x)$ and so to increase the fixation probability $u(x)=1-u^{*}(x)$. One can show that $u^{*}(x)$ satisfies the backward equation similar to Eq.(8) except for $k^{+}(x)$, with the boundary conditions $u^{*}(0)=1$ and $u^{*}(1)=0$. After replacing $u^{*}(x)$ by $u(x)=1-u^{*}(x)$, we obtain

$$
\frac{\sigma^{2}(x)}{2} \frac{d^{2} u}{d x^{2}}+\mu(x) \frac{d u}{d x}-k^{+}(x) u+k^{+}(x)=0
$$

Finally, we derive the backward equation under the joint effects of two killing functions under the assumption that the negative and positive background selections are two independent mechanisms. It follows that the Kolmogrov backward equation for $u(x)$ can be formulated by combining Eqs.(8) and Eq.(9), resulting in Eq.(1).

\section{Intra-population genetic diversity}

Let $J(x)=E[2 p(1-p)]$ be the expected heterozygosity of a nucleotide site. Under the standard steady-flux model, it is known that $J(x)$ satisfies the following backward equation

$$
\frac{\sigma^{2}(x)}{2} \frac{d^{2} J}{d x^{2}}+\mu(x) \frac{d J}{d x}+2 x(1-x) v=0
$$

Since both killing functions tend to reduce the genetic diversity by increasing the chance of a mutation to be either fixed or lost, the sum of two killing functions, $k^{+}(x)+k^{-}(x)$, can be considered as a single combined killing function. According to Karlin and Taylor (42), we obtain Eq.(2).

Derivation of Eq.(3) and Eq.(4) 
Under the selectively neutral model with two killing functions, we have $\mu(x)=0$, $\sigma^{2}=x(1-x) / 2 N_{e}, k^{-}(x)=b x(1-x)$, and $k^{+}(x)=h x(1-x)$. Eq.(1) can be simplified as follows

$$
\frac{d^{2} u}{d x^{2}}-(B+H) u+H=0
$$

where $B=4 N_{e} b, H=4 N_{e} h$ and $G=B+H$. The general solution of Eq.(11) can be written as $u(x)=C_{1} e^{Z_{1} x}+C_{2} e^{Z_{2} x}+H / G$, where $Z_{1}=G^{1 / 2}$ and $Z_{2}=-G^{1 / 2}$ and constants $C_{1}$ and $C_{2}$ are determined by the boundary conditions $u(0)=0$ and $u(1)=1$. When $x$ is small, it is convenient to use the approximations $\exp \left(Z_{1} x\right) \approx 1+Z_{1} x$ and $\exp \left(Z_{2} x\right) \approx 1+Z_{2} x$, respectively. After $u(x)$ is obtained, it is straightforward to have Eq.(3), with a new parameter $\beta=H / G$. In the same manner, under the neutral model, Eq.(4) can be simplified as follows

$$
\frac{d^{2} J}{d x^{2}}-(B+H) J+2 v=0
$$

which can be easily solved under the conditions of $J(0)=J(1)=0$.

\section{Statistical evaluation of parameter estimation}

The $95 \%$ confidence intervals (CI) for estimated $G$ and $\beta$ from Drosophila genome dataset can be approximately determined as follows. Based on the $95 \%$ CIs for synonymous diversities and distances provided by the original authors (27), we simulated a joint sampling density of these measures under the normal assumption, which can be used to empirically determine the $95 \%$ CIs of the estimates $G$ and $\beta$.

Acknowledges: The author is grateful to all members of my research group for constructive comments in the early version of this manuscript.

\section{Reference}

1. Hill WG \& Robertson A (1966) The effect of linkage on limits to artificial selection. Genet Res 8(3):269-294.

2. Felsenstein J (1974) The evolutionary advantage of recombination. Genetics 78(2):737-756. 
3. Smith JM \& Haigh J (1974) The hitch-hiking effect of a favourable gene. Genet Res 23(1):23-35.

4. Berry AJ, Ajioka JW, \& Kreitman M (1991) Lack of polymorphism on the Drosophila fourth chromosome resulting from selection. Genetics 129(4):11111117.

5. Charlesworth B, Morgan MT, \& Charlesworth D (1993) The Effect of Deleterious Mutations on Neutral Molecular Variation. Genetics 134(4):1289-1303.

6. Barton NH (2010) Genetic linkage and natural selection. Philosophical transactions of the Royal Society of London. Series B, Biological sciences 365(1552):2559-2569.

7. Stephan W (2010) Genetic hitchhiking versus background selection: the controversy and its implications. Philosophical transactions of the Royal Society of London. Series B, Biological sciences 365(1544):1245-1253.

8. Charlesworth B (2012) The Effects of Deleterious Mutations on Evolution at Linked Sites. Genetics 190(1):5-22.

9. Neher RA (2013) Genetic Draft, Selective Interference, and Population Genetics of Rapid Adaptation. Annu Rev Ecol Evol S 44:195-215.

10. Cutter AD \& Payseur BA (2013) Genomic signatures of selection at linked sites: unifying the disparity among species. Nat Rev Genet 14(4):262-274.

11. Stephan W (2019) Selective Sweeps. Genetics 211(1):5-13.

12. Kern AD \& Hahn MW (2018) The Neutral Theory in Light of Natural Selection. Mol Biol Evol 35(6):1366-1371.

13. Jensen JD, et al. (2019) The importance of the Neutral Theory in 1968 and 50 years on: A response to Kern and Hahn 2018. Evolution; international journal of organic evolution 73(1):111-114.

14. Begun DJ \& Aquadro CF (1992) Levels of Naturally-Occurring DNA Polymorphism Correlate with Recombination Rates in Drosophila-Melanogaster. Nature 356(6369):519-520.

15. Desai MM \& Fisher DS (2007) Beneficial mutation-selection balance and the effect of linkage on positive selection. Genetics 176(3):1759-1798.

16. Kaplan NL, Hudson RR, \& Langley CH (1989) The Hitchhiking Effect Revisited. Genetics 123(4):887-899.

17. Wiehe THE \& Stephan W (1993) Analysis of a Genetic Hitchhiking Model, and Its Application to DNA Polymorphism Data from Drosophila-Melanogaster. Mol Biol Evol 10(4):842-854.

18. Kim Y \& Stephan W (2000) Joint effects of genetic hitchhiking and background selection on neutral variation. Genetics 155(3):1415-1427.

19. Sella G, Petrov DA, Przeworski M, \& Andolfatto P (2009) Pervasive natural selection in the Drosophila genome? Plos Genet 5(6):e1000495.

20. Zeng K \& Charlesworth B (2011) The Joint Effects of Background Selection and Genetic Recombination on Local Gene Genealogies. Genetics 189(1):251-U940.

21. Coop G \& Ralph P (2012) Patterns of Neutral Diversity Under General Models of Selective Sweeps. Genetics 192(1):205-U438.

22. Vitti JJ, Grossman SR, \& Sabeti PC (2013) Detecting Natural Selection in Genomic Data. Annual Review of Genetics, Vol 47 47:97-120. 
23. Hermisson J \& Pennings PS (2017) Soft sweeps and beyond: understanding the patterns and probabilities of selection footprints under rapid adaptation. Methods Ecol Evol 8(6):700-716.

24. Nachman MW (2001) Single nucleotide polymorphisms and recombination rate in humans. Trends Genet 17(9):481-485.

25. Hellmann I, Ebersberger I, Ptak SE, Paabo S, \& Przeworski M (2003) A neutral explanation for the correlation of diversity with recombination rates in humans. American journal of human genetics 72(6):1527-1535.

26. Lohmueller KE, et al. (2011) Natural Selection Affects Multiple Aspects of Genetic Variation at Putatively Neutral Sites across the Human Genome. Plos Genet 7(10).

27. Campos JL, Halligan DL, Haddrill PR, \& Charlesworth B (2014) The Relation between Recombination Rate and Patterns of Molecular Evolution and Variation in Drosophila melanogaster. Mol Biol Evol 31(4):1010-1028.

28. Hartfield M \& Otto SP (2011) Recombination and hitchhiking of deleterious alleles. Evolution; international journal of organic evolution 65(9):2421-2434.

29. Kaiser VB \& Charlesworth B (2009) The effects of deleterious mutations on evolution in non-recombining genomes. Trends Genet 25(1):9-12.

30. Corbett-Detig RB, Hartl DL, \& Sackton TB (2015) Natural Selection Constrains Neutral Diversity across A Wide Range of Species. Plos Biol 13(4).

31. Elyashiv E, et al. (2016) A Genomic Map of the Effects of Linked Selection in Drosophila. Plos Genet 12(8).

32. Campos JL, Zhao L, \& Charlesworth B (2017) Estimating the parameters of background selection and selective sweeps in Drosophila in the presence of gene conversion. P Natl Acad Sci USA 114(24):E4762-E4771.

33. Pouyet F, Aeschbacher S, Thiery A, \& Excoffier L (2018) Background selection and biased gene conversion affect more than $95 \%$ of the human genome and bias demograpic inferences. Elife 7.

34. Rettelbach A, Nater A, \& Ellegren H (2019) How Linked Selection Shapes the Diversity Landscape in Ficedula Flycatchers. Genetics 212(1):277-285.

35. Stephan W, Wiehe THE, \& Lenz MW (1992) The Effect of Strongly Selected Substitutions on Neutral Polymorphism - Analytical Results Based on DiffusionTheory. Theor Popul Biol 41(2):237-254.

36. Durrett R \& Schweinsberg J (2004) Approximating selective sweeps. Theor Popul Biol 66(2):129-138.

37. Kim Y \& Stephan W (2003) Selective sweeps in the presence of interference among partially linked loci. Genetics 164(1):389-398.

38. Kim Y (2006) Allele frequency distribution under recurrent selective sweeps. Genetics 172(3):1967-1978.

39. Nicolaisen LE \& Desai MM (2013) Distortions in Genealogies due to Purifying Selection and Recombination. Genetics 195(1):221-+.

40. Martin G \& Lambert A (2015) A simple, semi-deterministic approximation to the distribution of selective sweeps in large populations. Theor Popul Biol 101:40-46.

41. Campos JL \& Charlesworth B (2019) The Effects on Neutral Variability of Recurrent Selective Sweeps and Background Selection. Genetics 212(1):287-303. 
42. Karlin S \& Taylor HM (1981) A second course in stochastic processes (Academic Press, New York) pp xviii, 542 p.

43. Du JC, et al. (2012) Pericentromeric Effects Shape the Patterns of Divergence, Retention, and Expression of Duplicated Genes in the Paleopolyploid Soybean. Plant Cell 24(1):21-32.

44. Peck JR (1994) A Ruby in the Rubbish - Beneficial Mutations, Deleterious Mutations and the Evolution of Sex. Genetics 137(2):597-606.

45. Hudson RR \& Kaplan NL (1995) Deleterious Background Selection with Recombination. Genetics 141(4):1605-1617.

46. Nordborg M, Charlesworth B, \& Charlesworth D (1996) The effect of recombination on background selection. Genet Res 67(2):159-174.

47. Stephan W, Charlesworth B, \& McVean G (1999) The effect of background selection at a single locus on weakly selected, partially linked variants. Genet Res 73(2):133-146.

48. Eyre-Walker A \& Keightley PD (2007) The distribution of fitness effects of new mutations. Nat Rev Genet 8(8):610-618.

49. McVicker G, Gordon D, Davis C, \& Green P (2009) Widespread genomic signatures of natural selection in hominid evolution. Plos Genet 5(5):e1000471.

50. Kimura M (1969) The number of heterozygous nucleotide sites maintained in a finite population due to steady flux of mutations. Genetics 61(4):893-903.

51. McVean GAT \& Charlesworth B (2000) The effects of Hill-Robertson interference between weakly selected mutations on patterns of molecular evolution and variation. Genetics 155(2):929-944.

52. Charlesworth B (2012) The Role of Background Selection in Shaping Patterns of Molecular Evolution and Variation: Evidence from Variability on the Drosophila X Chromosome. Genetics 191(1):233-246.

53. Charlesworth B \& Campos JL (2014) The Relations Between Recombination Rate and Patterns of Molecular Variation and Evolution in Drosophila. Annu Rev Genet 48:383-403.

54. Kimura M (1983) The neutral theory of molecular evolution (Cambridge University Press, Cambridge Cambridgeshire ; New York) pp xv, 367 p.

55. Comeron JM (2014) Background Selection as Baseline for Nucleotide Variation across the Drosophila Genome. Plos Genet 10(6).

56. Hwang EY, et al. (2019) Genetic Diversity and Phylogenetic Relationships of Annual and Perennial Glycine Species. G3 9(7):2325-2336.

57. Gordo I, Navarro A, \& Charlesworth B (2002) Muller's ratchet and the pattern of variation at a neutral locus. Genetics 161(2):835-848.

58. Arbeithuber B, Betancourt AJ, Ebner T, \& Tiemann-Boege I (2015) Crossovers are associated with mutation and biased gene conversion at recombination hotspots. P Natl Acad Sci USA 112(7):2109-2114.

59. Miller DE, et al. (2016) Whole-Genome Analysis of Individual Meiotic Events in Drosophila melanogaster Reveals That Noncrossover Gene Conversions Are Insensitive to Interference and the Centromere Effect. Genetics 203(1):159-171.

60. Messer PW (2013) SLiM: simulating evolution with selection and linkage. Genetics 194(4):1037-1039. 
bioRxiv preprint doi: https://doi.org/10.1101/2020.01.11.901066; this version posted January 14,2020 . The copyright holder for this preprint (which was not certified by peer review) is the author/funder, who has granted bioRxiv a license to display the preprint in perpetuity. It is made available under aCC-BY-NC-ND 4.0 International license.

61. Messer PW \& Petrov DA (2013) Frequent adaptation and the McDonaldKreitman test. Proc Natl Acad Sci U S A 110(21):8615-8620. 


\section{Figure legends}

Fig.1. (A) The ratio of neutral evolutionary rate to mutation rate $(\lambda / v)$ plotted against the strength $(G)$ of generalized background selection, given the proportion of positive background selection $\beta=0,0.05,0.1,0.2,0.5$ or 1.0 , respectively. Note that there exists a critical value $\beta_{c} \approx 0.334$. When $\beta<\beta_{c}$, the ratio $\lambda / v$ decreases toward the area of $\lambda / v<1$ with the increasing of $G$ until approaching to a minimum; since then $\lambda / v$ increases with the increasing of $G$, ultimately toward the area of $\lambda / \nu>1$. When $\beta>\beta_{c}$, the ratio $\lambda / v$ increases with the increase of $G$ such that $\lambda / v>1$ holds always. (B) The ratio of neutral genetic diversity $\left(\pi / \pi_{0}\right)$ plotted against the strength $(G)$ of generalized background selection. Here $\pi$ is the expected neutral intra-population diversity under the generalized background selection, and $\pi_{0}$ is that with no generalized background selection.

Fig.2. The $\lambda / v-\pi / \pi_{0}$ curve, while the proportion of positive background selection $\beta=0$, $0.005,0.01,0.05,0.1,0.2,0.5$ and 1.0 , respectively.

Fig.3. Analysis of divergence and variation in human chromosome regions with different recombination rate ( $r$, measured by cM/Mb); data from Nachman (24). (A) The mean strength of generalized background selection $(G)$ in genes located in low $(\mathrm{cM} / \mathrm{Mb}<1)$, middle $(1<\mathrm{cM} / \mathrm{Mb}<2)$ and high $(\mathrm{cM} / \mathrm{Mb}>2)$ recombination regions, respectively, as well as that of all genes. Standard errors are presented. (B) The mean proportion of positive background selection in genes located in low, middle and high recombination regions, respectively, as well as that of all genes. Standard errors are presented. (C) The squared root of $G$ estimates plotted against the inverse of $r$, the recombination rate. The coefficient of correlation is $0.73, P$-value $<0.01$. 


\section{Tables}

Table 1. A summary for the conceptual framework of the generalized background selection

\begin{tabular}{|c|c|c|}
\hline $\begin{array}{c}\text { Types of generalized } \\
\text { background selections }\end{array}$ & $\begin{array}{c}\text { Negative background } \\
\text { selection }\end{array}$ & $\begin{array}{c}\text { Positive background } \\
\text { selection }\end{array}$ \\
\hline Examples & $\begin{array}{l}\text { purifying selection at } \\
\text { closely-linked sites }\end{array}$ & $\begin{array}{l}\text { positive selection at } \\
\text { closely-linked sites } \\
\text { (selective sweep) }\end{array}$ \\
\hline Within population & $\begin{array}{l}\text { reduction of genetic } \\
\text { variation }\end{array}$ & $\begin{array}{l}\text { reduction of genetic } \\
\text { variation }\end{array}$ \\
\hline Between species & $\begin{array}{l}\text { decrease the rate of } \\
\text { sequence evolution }\end{array}$ & $\begin{array}{l}\text { increase the rate of } \\
\text { sequence evolution }\end{array}$ \\
\hline Killing functions & $\begin{array}{c}k(x) \text { : decrease the fixation } \\
\text { probability of a mutation }\end{array}$ & $\begin{array}{l}k^{+}(x) \text { : decrease the loss } \\
\text { probability of a mutation }\end{array}$ \\
\hline $\begin{array}{l}\text { Strength of generalized } \\
\text { background selection } \\
\qquad(G=B+H)\end{array}$ & $\begin{array}{l}B \text { : Strength of negative } \\
\text { background selection }\end{array}$ & $\begin{array}{l}H \text { : Strength of positive } \\
\text { background selection }\end{array}$ \\
\hline Relative contributions & $1-\beta=B /(B+H)$ & $\beta=H /(B+H)$ \\
\hline
\end{tabular}


Table 2. Statistical analysis of Drosophila population genomics data ${ }^{a}$.

\begin{tabular}{|c|c|c|c|c|}
\hline NC regions ${ }^{c}$ & $\pi_{s} / \pi_{0}$ & $d_{s} / d_{s 0}$ & $G$ & $\beta(\%)$ \\
\hline \multicolumn{5}{|c|}{ Crossover regions: $\mathrm{AC}^{b}$ (7099 genes) } \\
\hline N2 (59 genes) & $\begin{array}{c}0.157 \\
(0,0.314)^{d}\end{array}$ & $\begin{array}{c}1.122 \\
(1.062,1.183)\end{array}$ & $\begin{array}{c}1.63 \times 10^{2} \\
\left(0.41 \times 10^{2}, \infty\right)\end{array}$ & $\begin{array}{c}8.79 \\
(0,15.6)\end{array}$ \\
\hline N3 (99 genes) & $\begin{array}{c}0.116 \\
(0,0.232)\end{array}$ & $\begin{array}{c}1.084 \\
(1.042,1.130)\end{array}$ & $\begin{array}{c}2.99 \times 10^{2} \\
\left(0.75 \times 10^{2}, \infty\right)\end{array}$ & $\begin{array}{c}6.27 \\
(0,11.5)\end{array}$ \\
\hline N4 (67 genes) & $\begin{array}{c}0.057 \\
(0,0.115)\end{array}$ & $\begin{array}{c}0.947 \\
(0.908,0.990)\end{array}$ & $\begin{array}{c}12.2 \times 10^{2} \\
\left(0.77 \times 10^{2}, \infty\right)\end{array}$ & $\begin{array}{c}2.71 \\
(0,11.4)\end{array}$ \\
\hline NXc (19 genes) & $\begin{array}{c}0.315 \\
(0,0.633)\end{array}$ & $\begin{array}{c}0.962 \\
(0.863,1.057)\end{array}$ & $\begin{array}{c}0.404 \times 10^{2} \\
\left(0.10 \times 10^{2}, \infty\right)\end{array}$ & $\begin{array}{c}15.1 \\
(0,31.6)\end{array}$ \\
\hline NXt (23 genes) & $\begin{array}{c}0.129 \\
(0,0.263)\end{array}$ & $\begin{array}{c}0.969 \\
(0.893,1.046)\end{array}$ & $\begin{array}{c}2.38 \times 10^{2} \\
\left(0.58 \times 10^{2}, \infty\right)\end{array}$ & $\begin{array}{c}6.29 \\
(0,13.1)\end{array}$ \\
\hline Pooled (268 genes) & $\begin{array}{c}0.155 \\
(0.071,0.240)\end{array}$ & $\begin{array}{c}1.042 \\
(1.015,1.065)\end{array}$ & $\begin{array}{c}1.67 \times 10^{2} \\
\left(0.70 \times 10^{2}, 8.11 \times 10^{2}\right)\end{array}$ & $\begin{array}{c}8.06 \\
(3.51,12.0)\end{array}$ \\
\hline \multicolumn{5}{|c|}{ Crossover regions: $\mathrm{XC}^{b}$ (1319 genes) } \\
\hline N2 (59) & $\begin{array}{c}0.142 \\
(0,0.284)\end{array}$ & $\begin{array}{c}1.140 \\
(1.078,1.202)\end{array}$ & $\begin{array}{c}1.99 \times 10^{2} \\
\left(0.45 \times 10^{2}, \infty\right)\end{array}$ & $\begin{array}{c}8.09 \\
(0,14.3)\end{array}$ \\
\hline N3 (99) & $\begin{array}{c}0.104 \\
(0,0.209)\end{array}$ & $\begin{array}{c}1.101 \\
(1.058,1.147)\end{array}$ & $\begin{array}{c}3.66 \times 10^{2} \\
\left(1.41 \times 10^{2}, \infty\right)\end{array}$ & $\begin{array}{c}5.75 \\
(0,9.63)\end{array}$ \\
\hline N4 (67) & $\begin{array}{c}0.052 \\
(0,0.104)\end{array}$ & $\begin{array}{c}0.961 \\
(0.922,1.004)\end{array}$ & $\begin{array}{c}14.9 \times 10^{2} \\
\left(0.92 \times 10^{2}, \infty\right)\end{array}$ & $\begin{array}{c}2.49 \\
(0,10.6)\end{array}$ \\
\hline NXc (19) & $\begin{array}{c}0.285 \\
(0,0.571)\end{array}$ & $\begin{array}{c}0.977 \\
(0.877,1.074)\end{array}$ & $\begin{array}{c}0.494 \times 10^{2} \\
\left(0.13 \times 10^{2}, \infty\right)\end{array}$ & $\begin{array}{c}13.9 \\
(0,25.7)\end{array}$ \\
\hline NXt (23) & $\begin{array}{c}0.117 \\
(0,0.237)\end{array}$ & $\begin{array}{c}0.984 \\
(0.907,1.062)\end{array}$ & $\begin{array}{c}2.91 \times 10^{2} \\
\left(0.61 \times 10^{2}, \infty\right)\end{array}$ & $\begin{array}{c}5.77 \\
(0,11.9)\end{array}$ \\
\hline Pooled (268) & $\begin{array}{c}0.140 \\
(0.099,0.217)\end{array}$ & $\begin{array}{c}1.058 \\
(1.031,1.081)\end{array}$ & $\begin{array}{c}2.05 \times 10^{2} \\
\left(0.94 \times 10^{2}, \infty\right)\end{array}$ & $\begin{array}{c}7.39 \\
(3.20,10.2)\end{array}$ \\
\hline
\end{tabular}

Note: $a$. data from Campos et al. (27). $b$. AC short for autosome crossover regions, and XC for Xchromosome regions. $c$. five non-crossover regions and the pooled. $d$. the quantities in parentheses are the $95 \%$ CIs of the estimates, under a normal approximation. 
bioRxiv preprint doi: https://doi.org/10.1101/2020.01.11.901066; this version posted January 14,2020 . The copyright holder for this preprint (which was not certified by peer review) is the author/funder, who has granted bioRxiv a license to display the preprint in perpetuity. It is made available under aCC-BY-NC-ND 4.0 International license.

\section{Figures}

Figure 1

(A)

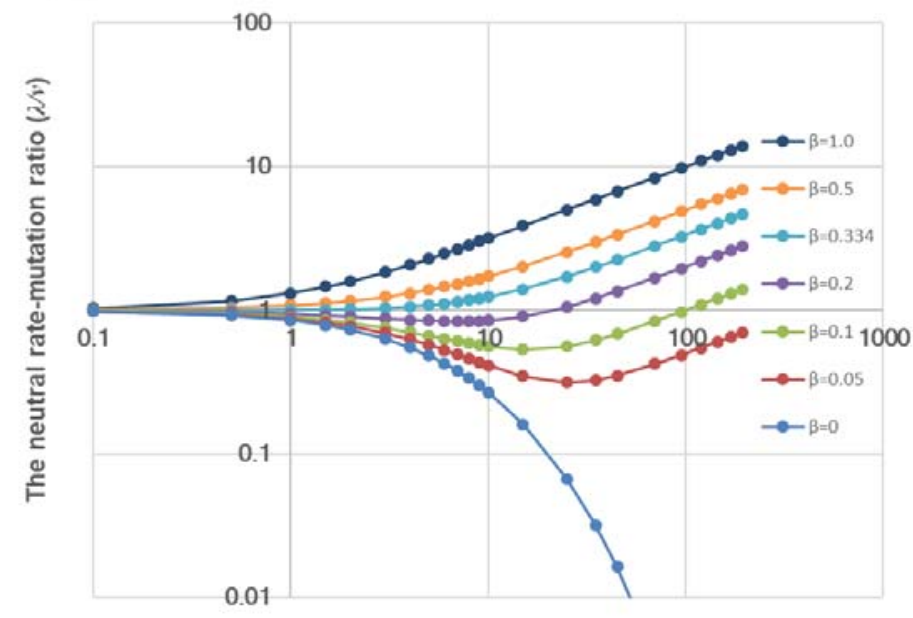

Strength of generalized background selection $(G)$

(B)

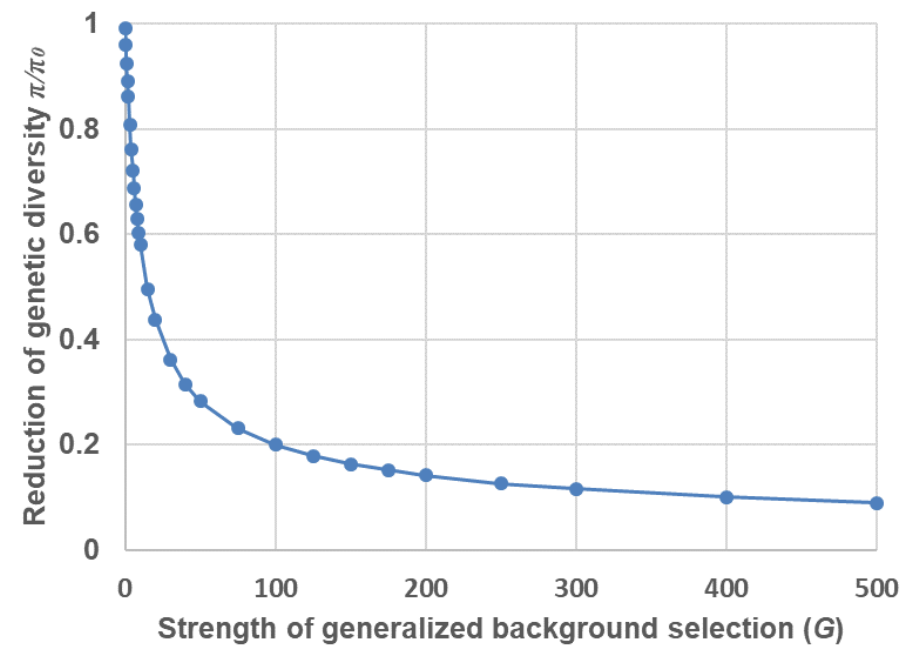


bioRxiv preprint doi: https://doi.org/10.1101/2020.01.11.901066; this version posted January 14,2020. The copyright holder for this preprint (which was not certified by peer review) is the author/funder, who has granted bioRxiv a license to display the preprint in perpetuity. It is made available under aCC-BY-NC-ND 4.0 International license.

Figure 2

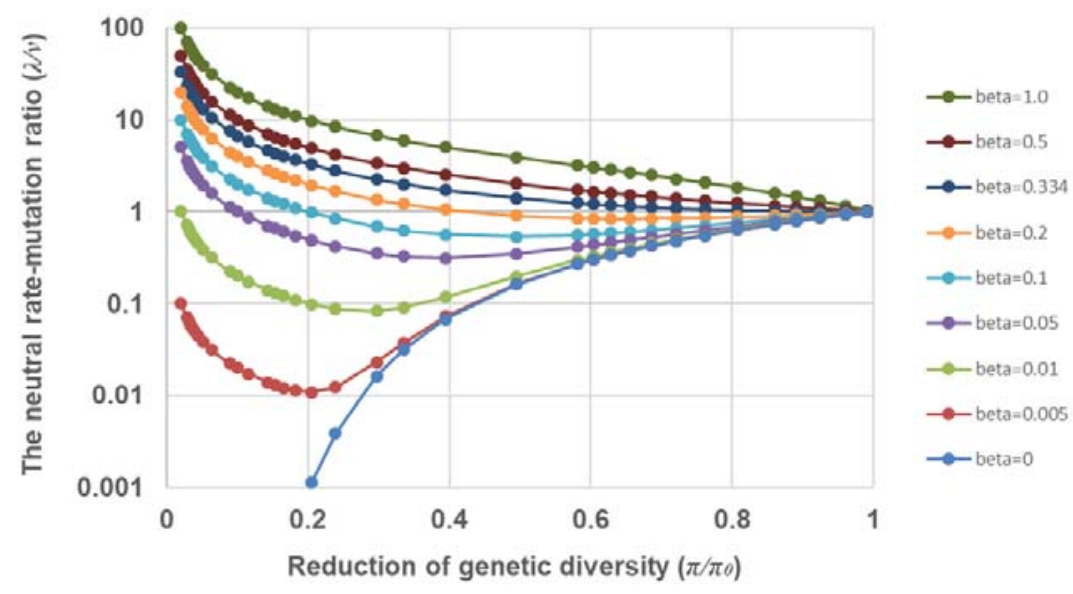


bioRxiv preprint doi: https://doi.org/10.1101/2020.01.11.901066; this version posted January 14,2020 . The copyright holder for this preprint (which was not certified by peer review) is the author/funder, who has granted bioRxiv a license to display the preprint in perpetuity. It is made available under aCC-BY-NC-ND 4.0 International license.

Figure 3

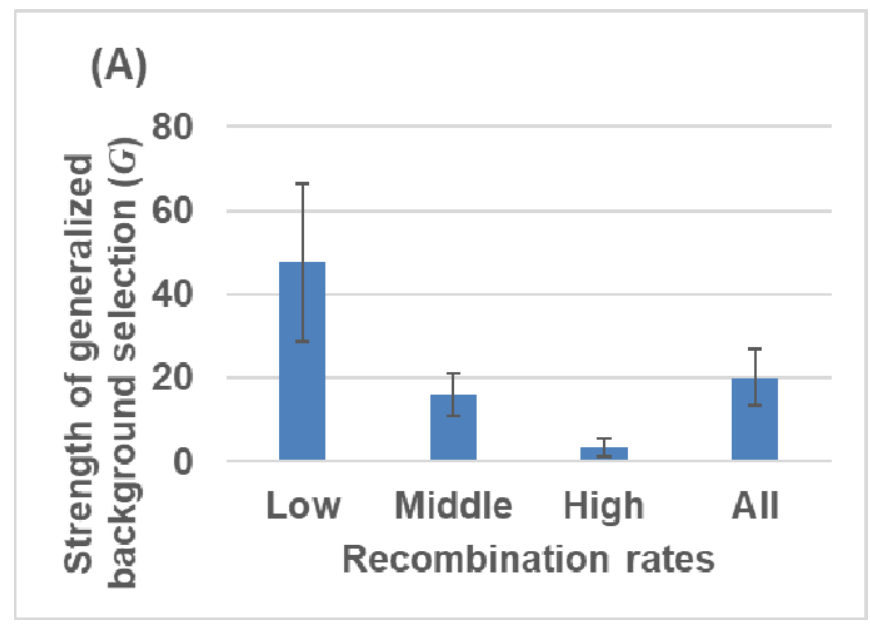

(B)

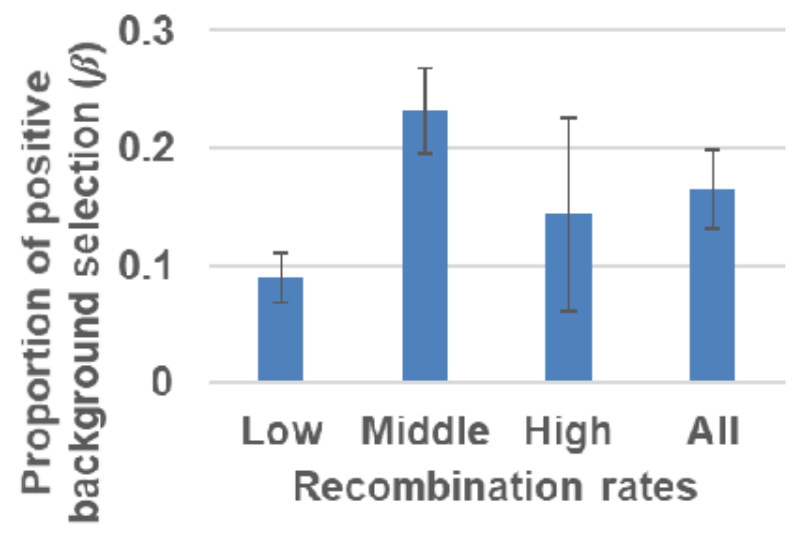

(C)

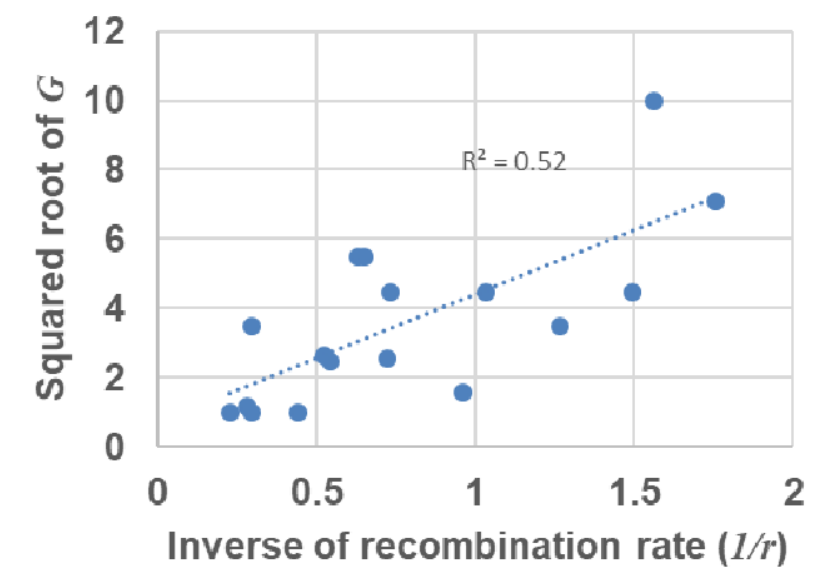

